\title{
CISTECTOMÍA RADICAL LAPAROSCÓPICA EN LOS TUMORES VESICALES CLÍNICAMENTE LOCALIZADOS (T2)
}

\author{
Lluís Cecchini Rosell, Anna Orsola de los Santos, Carles X. Raventós Busquets, Enrique Trilla \\ Herrera, Jacques Planas Morin, Ana Celma Doménech, Carlos Salvador Lacambra y Juan \\ Morote Robles.
}

Servicio de Urología. Hospital Universitario Vall d’Hebron. Barcelona. España.

\begin{abstract}
Resumen.- OBJETIVO: La cistectomía radical laparoscópica se ha desarrollado a partir de la expansión de la prostatectomía radical laparoscópica. Esta técnica permite un abordaje poco invasivo para el tratamiento de los tumores vesicales infiltrantes de la capa muscular con disminución del sangrado y una más rápida recuperación postoperatoria.
\end{abstract}

MÉTODOS: Entre septiembre de 2004 y enero de 2007 se han realizado 54 cistectomías radicales por vía laparoscópica, 48 en estadio T2 y de estas últimas 43 (90\%) eran varones y 5 (10\%) mujeres. La edad media fue de 64 años (27-88a). La linfadenectomía se practicó por acceso laparoscópico en todos los casos, obteniendo una media de 13 ganglios (4-24). La derivación urinaria se realizó por la incisión de extracción del espécimen en todos los casos menos uno que se realizó completamente intracorpóreo, siendo ureteroileostomía cutánea tipo Bricker en 30 casos (62\%), neovejiga ortotópica tipo Padovana en 17 casos (35\%) y ureterostomía cutánea en un caso (2\%).

RESULTADOS: El tiempo quirúrgico medio de todo el procedimiento fue de 287 minutos (180-480), $270 \mathrm{mi}$ nutos para los casos con derivación tipo Bricker y de 316 para los casos con una neovejiga. El índice de transfusión fue del 25\%. El tiempo medio de íleo paralitico fue de 5 días (2-10d) con un tiempo medio de ingreso para los pacientes con Bricker de 13 días (6-34) y de 16 días (8-30) para las neovejigas. El control oncológico, con un seguimiento medio de 10,8 meses $10,4-$ $30 \mathrm{~m})$, presenta una supervivencia cáncer específica del $90 \%$ con un tiempo medio de supervivencia estimado de 28 meses (IC 95\% 26-30). La supervivencia media global ha sido del $79 \%$ con un tiempo de supervivencia de 26 meses (IC 95\% 23-29).

CONCLUSIONES: La cistectomía radical laparoscópica es una técnica factible que ofrece ventajas. Permite una exéresis con un menor sangrado y un postoperatorio más llevadero. Estudios aleatorizados deberían demostrar estas ventajas para confirmar si puede llegar a ser la técnica de elección. La realización de la derivación urinaria por la laparotomía, obligada para la extracción de la pieza quirúrgica, optimiza los resultados de la derivación y el tiempo quirúrgico total sin reducir los beneficios de la exéresis laparoscópica.

Palabras clave: Cistectomía. Cáncer vesical. Laparoscopica.

Summary.- OBJECTIVES: Laparoscopic radical cystectomy has been developed after the expansion of laparoscopic radical prostatectomy. This technique makes possible a minimally invasive approach to muscle-invasive bladder cancer with less blood loss and faster postoperative recovery. 
METHODS: From September 2004 to January 2007, 54 laparoscopic radical cystectomies were performed, 48 of them in stage 12 , from which $43(90 \%)$ were male and $5(10 \%)$ female patients. Mean age was 64 years $(27$ 88). Lymphadenectomy was carried out by laparoscopic approach in all cases, with a mean of 13 nodes obtained (4-24). Urinary diversion was done through the incision needed to extract the specimen in all cases but one that was completed completely intracorporeally; constructing a Bricker-type ureteroileostomy in 30 (62\%) cases, orthotopic neobladder (Vesica lleale Padovana) in 17 cases (35\%), and cutaneous ureterostomy in 1 case $(2 \%)$.

RESULTS: Mean surgical time for the whole procedure was 287 minutes (180-480), 270 minutes for Brickertype derivation cases and 316 minutes for neobladder cases. Blood transfusion rate was 25\%. Mean ileal paralysis was 5 days (2-10) with a mean hospital stay of 13 days (6-34) for Bricker cases and 16 days (8-30) for neobladder cases. Oncological control, after a mean follow-up of 10,8 months (0,4-30), showed a cancerspecific survival of $90 \%$ with a mean survival time of 28 months (95\% Cl 26-30). Global mean survival was 79\% with a mean survival of 26 months (95\% Cl 23-29).

CONCLUSIONS: Laparoscopic radical cystectomy is a feasible technique that offers some advantages. It allows excision with less blood loss and an easier postoperative period. Randomized studies should demonstrate these advantages to confirm this approach as the technique of choice. Urinary diversion performed through the laparotomy incision, necessary to extract the specimen, optimizes derivation results and whole surgical time without reducing the beneficial effects of the laparoscopic exeresis.

Keywords: Cystectomy. Bladder cancer. Laparoscopy.

\section{INTRODUCCIÓN}

La cistectomía realizada por un abordaje laparoscópico, aunque inicialmente fue descrita en los años noventa $(1,2)$, se ha desarrollado como técnica después de la generalización de la prostatectomía radical laparoscópica. El conocimiento anatómico de la pelvis que ha supuesto la difusión de esa técnica ha permitido ampliar su aplicación a la cistectomía radical. Maniobras como la disección de las vesículas seminales a nivel del fondo de saco de Douglas o la separación del espacio de Retzius para el abordaje anterior en la próstata permitieron imaginar cómo sería proceder a la exéresis completa de la vejiga. Así para muchos urólogos con experiencia laparoscópica, la cistectomía radical resultó el paso lógico en la incorporación de nuevas técnicas de forma más o menos simultánea en diversos centros.
Esta alternativa a la cistectomía abierta ha ido ganando cada vez más aceptación, aunque todavía no se ha consolidado para el tratamiento del cáncer vesical. Existen varios puntos de controversia incluso entre los defensores de este abordaje. Entre ellos cabe destacar el efecto condicionante del abordaje laparoscópico en la extensión de la linfadenectomía (3) o la utilidad de la creación intracorpórea de la derivación urinaria (4). Otro punto controvertido es el manejo del muñón uretral para evitar el riesgo oncológico que supone el derrame de orina potencialmente depositaria de células transicionales $(5,6)$. Existe también discusión sobre el uso de esta técnica para el tratamiento de tumores localmente avanzados, que ya ofrecen malos resultados sea cual sea el manejo quirúrgico (7).

En esta revisión analizamos estos puntos, junto con los conceptos ya establecidos, y presentamos los resultados de nuestra serie en aquellos casos de tumores clínicamente localizados.

\section{PACIENTES Y MÉTODOS}

Desde septiembre de 2004 hemos realizado 54 cistectomías radicales por laparoscopia. Se ha ofrecido esta técnica quirúrgica a todos los pacientes candidatos a cistectomía sin excluir a ninguno por edad, cirugías previas, obesidad o tumores con extensión local (>T2). En nuestra unidad todas las indicaciones de cistectomía se abordan por laparoscopia, ya que consideramos que los beneficios potenciales son superiores a los inconvenientes que pueda suponer la laparoscopia. En este artículo presentamos los resultados para los tumores con estadios clínicos $<=$ T2 $(n=48)$, pues son el grupo sobre los que hay más consenso respecto al uso de este abordaje.

La técnica quirúrgica está ya descrita en la literatura por los autores $(8,9)$ y no difiere mucho de la publicada por otros grupos (10-12). El paciente se posiciona en decúbito supino con las piernas ligeramente en abducción para colocar la columna de laparoscopia en medio. Los soportes de hombro permiten usar la posición de Trendelemburg forzado $\left(30^{\circ}\right)$ necesaria para separar cranealmente los intestinos de la pelvis y presentar los límites anatómicos de la vejiga. Los brazos se colocan a lo largo del cuerpo, como en la prostatectomía radical laparoscópica, para no interferir en la posición del equipo quirúrgico. El trocar inicial lo colocamos por técnica de minilaparotomía (13) dos traveses de dedo por encima del ombligo.

Tras la exéresis de la pieza, se realiza la linfadenectomía iliobturatriz por laparoscopia, inclu- 
TABLA I. COMPLICACIONES POSTOPERATORIAS.

\begin{tabular}{|l|l|}
\hline Fiebre postoperatoria & $6(12)$ \\
\hline Dehiscencia sutura laparotomía & $3(6)$ \\
\hline Infección herida quirúrgica & $3(6)$ \\
\hline Fístula urinaria & $2(4)$ \\
\hline
\end{tabular}

yendo todo el tejido envolvente a los vasos iliacos externos y comunes. Se emplea como límite lateral el nervio genitofemoral y medial e inferiormente el nervio obturatriz, que deben identificarse. No suele haber dificultad en progresar hasta la zona de la ilíaca común por laparoscopia. Para la extensión craneal de la linfadenectomía, en caso de considerarla necesaria, pueden colocarse otros trocares auxiliares a la altura del ombligo o bien realizarse por la propia laparotomía de la derivación, llegando así a la zona sacra y de la bifurcación aórtica.

En cuanto a la reconstrucción urinaria, para la derivación tipo Bricker se amplia el trocar central hipogástrico simétricamente (caudal y cranealmente) hasta una incisión (entre 6 y $8 \mathrm{~cm}$ ) que permita la extracción del íleon y la anastomosis ureteral. En el caso de derivaciones ortotópicas, se modifica la incisión, ampliándola desde ese mismo trocar hacia el pubis, para permitir la anastomosis uretral bajo control visual. Este proceder se adoptó para ahorrar la realización de un segundo neumoperitoneo y acortar así el tiempo quirúrgico.

Los pacientes han sido seguidos periódicamente mediante analítica y TC de control cada 3 meses inicialmente y después cada 6 meses.

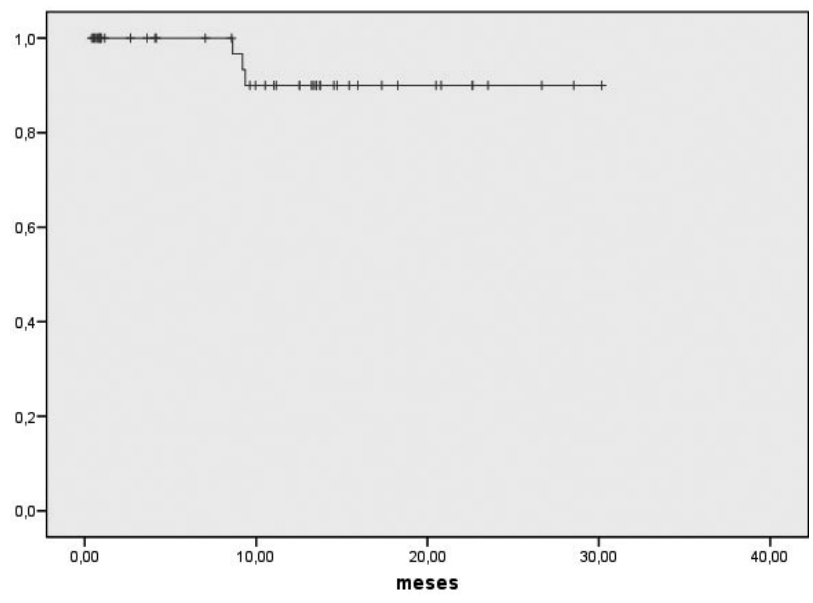

FIGURA 1. Supervivencia cáncer específica.

\section{RESULTADOS}

De los 48 pacientes con tumores vesicales estadio clínico T2, $43(90 \%)$ eran varones y $5(10 \%)$ mujeres, con una edad media de 64 años (27-88a). La derivación urinaria se ha realizado por la incisión de extracción de la pieza en todos los casos menos uno. En ese caso se realizó una sustitución vesical en una mujer tras la extracción de la pieza por vagina y en la que la neovejiga se realizó por completo mediante sutura intracorpórea. La derivación urinaria ha sido tipo Bricker en 30 casos (62\%), neovejiga tipo Padovana en 17 casos (35\%) y ureterostomía cutánea en un paciente monoreno izquierdo (2\%). La linfadenectomía se practicó por acceso laparoscópico en todos los casos, extendiéndose cranealmente cuando se consideró conveniente por la misma incisión de extracción de la pieza antes de realizar la derivación. Así se ha obtenido una media de 13 ganglios (4-24).

El tiempo quirúrgico medio de todo el procedimiento ha sido de 287 minutos (180-480 min; ds 63), siendo de 270 min para los casos con derivación tipo Bricker y de 316 para los casos con una neovejiga. El índice de transfusión ha sido del 25\%, con 2 concentrados de hematíes de media. El tiempo medio de íleo paralítico ha sido de 5 días (2-10 días) con un tiempo de ingreso para los pacientes con derivación tipo Bricker de 13 días (6-34 d) y de 16 días $(8-30$ d) para las derivaciones urinarias complejas.

Las complicaciones peroperatorias se detallan en la Tabla I. Destacan un $12 \%$ de casos de fiebre postoperatoria, con 2 casos de fugas urinarias y un $6 \%$ de problemas de la herida (infección y dehiscencia). Las histologías y las características tumorales se detallan en la Tabla II. Los márgenes quirúrgicos han sido todos negativos y no ha habido ninguna recidiva local.

Con un seguimiento medio de 10,8 meses $(0,4-30 \mathrm{~m})$, la supervivencia cáncer específica ha sido del $90 \%$ con un tiempo medio de supervivencia estimado de 28 meses (IC 95\% 26-30) (Figura 1). La supervivencia media global ha sido del $79 \%$ con un tiempo de supervivencia de 26 meses (IC 95\% 23-29) (Figura 2). No hemos hallado diferencias significativas, aunque sí una tendencia a mejor supervivencia de los T2 patológicos respecto a los T3 (Figura 3 ). En la actualidad el $75 \%$ de los pacientes están en situación estable mientras que un $12 \%$ están en situación de recidiva o progresión de la enfermedad. Seis pacientes han sido exitus (12\%), de los cuales 4 $(67 \%)$ lo han sido por causa tumoral y dos (33\%) por otras causas. 


\section{DISCUSIÓN}

En esta revisión de nuestra serie de cistectomías radicales laparoscópicas acotada a los T2 clínicos, demostramos que este abordaje es factible especialmente en estos casos. El tiempo quirúrgico medio de unas 4,5 horas y el número de ganglios obtenido supera el mínimo de 9 ganglios necesarios para un buen resultado oncológico (14). Esto se ha conseguido obteniendo además todos los márgenes negativos. Por otro lado, aunque no se trata de un estudio comparativo con la técnica abierta, la recuperación postoperatoria y el índice de transfusiones han mejorado nuestra propia experiencia con cirugía abierta. El índice de complicaciones peroperatorias es superponible al de la cirugía abierta.

El desarrollo de la cistectomía radical por vía laparoscópica ha nacido de forma espontánea en diversos grupos con experiencia contrastada en ese tipo de abordaje. Las distintas series publicadas $(4,10,15-18)$ y las más recientes ya con seguimiento oncológico superior a tres años $(19,20)$ muestran la rápida evolución de la técnica y su establecimiento. Entre los temas más debatidos destaca el de la realización laparoscópica de la derivación urinaria, aunque actualmente parece haberse alcanzado un

TABLA II.

\begin{tabular}{|l|c|}
\hline & $N(\%)$ \\
Carcinoma de células transicionales (CCT) & $27(56)$ \\
$9(19)$ \\
Carcinoma escamoso & $6(12)$ \\
Otras histologías & $6(12)$ \\
\hline pT0 & $7(15)$ \\
pTis & $2(4)$ \\
PT1 & $2(4)$ \\
pT2 & $17(35)$ \\
pT3 & $14(29)$ \\
pT4 & $6(12)$ \\
\hline CIS asociado & $23(48)$ \\
\hline pN0 & $36(75)$ \\
pN1 & $6(12)$ \\
pN2 & $6(12)$ \\
\hline MO & $48(100)$ \\
Márgenes afectos & $0(0)$ \\
\hline
\end{tabular}

consenso respecto a la poca utilidad de esta parte de la intervención. Como el tamaño del espécimen obliga en la mayoría de casos a una incisión abdominal, ésta suele ser de medida suficiente para extraer el intestino y realizar el aislamiento del asa ileal por vía abierta de forma más segura y rápida. Esas ventajas en tiempo y seguridad son aún más obvias cuando se plantea la creación de un reservorio para la sustitución vesical, pues la reconstrucción laparoscópica de un reservorio ortotópico mediante suturas manuales es de gran complejidad y alarga en exceso el tiempo quirúrgico. El abordaje completo por laparoscopia debería restringirse a aquellos casos en que la extracción de la pieza se pueda realizar sin laparotomía, por ejemplo por vía vaginal o rectal. De esta forma se pueden obtener excelentes resultados en la derivación sin alargar los tiempos quirúrgicos ni arriesgar complicaciones.

En cuanto a la linfadenectomía iliobturatriz, ésta es fácilmente abordable por laparoscopia después de la exéresis de la pieza, con los límites anatómicos bien definidos y sin la molestia de la pieza quirúrgica in situ. Algunos autores prefieren realizarla al inicio de la intervención para delimitar mejor los vasos que interesan en la posterior cistectomía (10). Otra opción es practicar la exéresis en bloque de la pieza de cistectomía con los ganglios. Sin embargo, esta extracción en bloque puede dificultar la cuantificación de los ganglios por parte del patólogo.

En nuestra experiencia la cistectomía laparoscópica supone además diversas ventajas sobre el abordaje clásico. Las ventajas del abordaje laparoscópico en la cistectomía son en parte superponibles a las de la prostatectomía radical: la mejor visión del fondo pélvico, el menor sangrado, la menor manipulación del intestino y el menor dolor postoperatorio. La visión que ofrece el laparoscopio es inmejorable

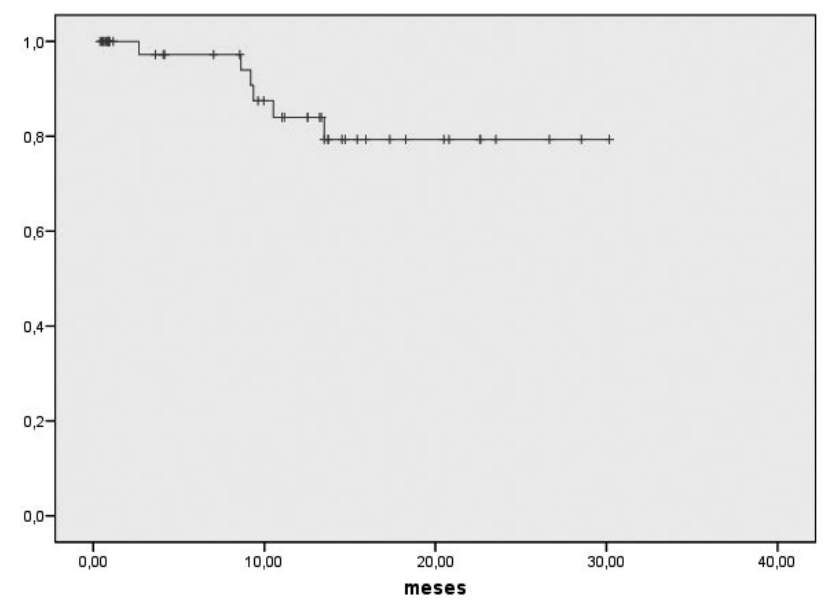

FIGURA 2. Supervivencia global. 
ya que permite acceder a la pelvis masculina, el género más frecuentemente afectado por esta patología, sin problemas de interferencias o falta de luminosidad. El menor sangrado se debe en parte al efecto hemostático de la presión del $\mathrm{CO}_{2}$ sobre el sangrado venoso (de baja presión) y en parte por la cuidadosa hemostasia que permite la visión con aumento y que además es necesaria para trabajar con una cámara. La suma de los dos factores revierte en un menor sangrado demostrado también en otras técnicas quirúrgicas laparoscópicas (21-24). La mayoría de las series demuestran una clara disminución del sangrado quirúrgico, que aún no se refleja claramente en el índice transfusional de nuestra serie. Estos pacientes suelen presentarse en una situación frágil y acumulando un estado anémico propio del tumor y de la intervención por resección transuretral reciente, por lo que hay un hábito histórico de transfundir perioperatoriamente (en quirófano y en la reanimación anestésica) a los pacientes sometidos a cistectomía convencional por vía abierta, que los anestesistas siguen aplicando a estos casos laparoscópicos.

Por otra parte, la evolución postoperatoria es más rápida, con menor dolor y una recuperación más precoz del tránsito intestinal. La manipulación intestinal, imprescindible en la técnica clásica para un buen abordaje de la zona pélvica, se minimiza gracias al posicionamiento en Trendelemburg y el propio neumoperitoneo. Además, al no mantener el

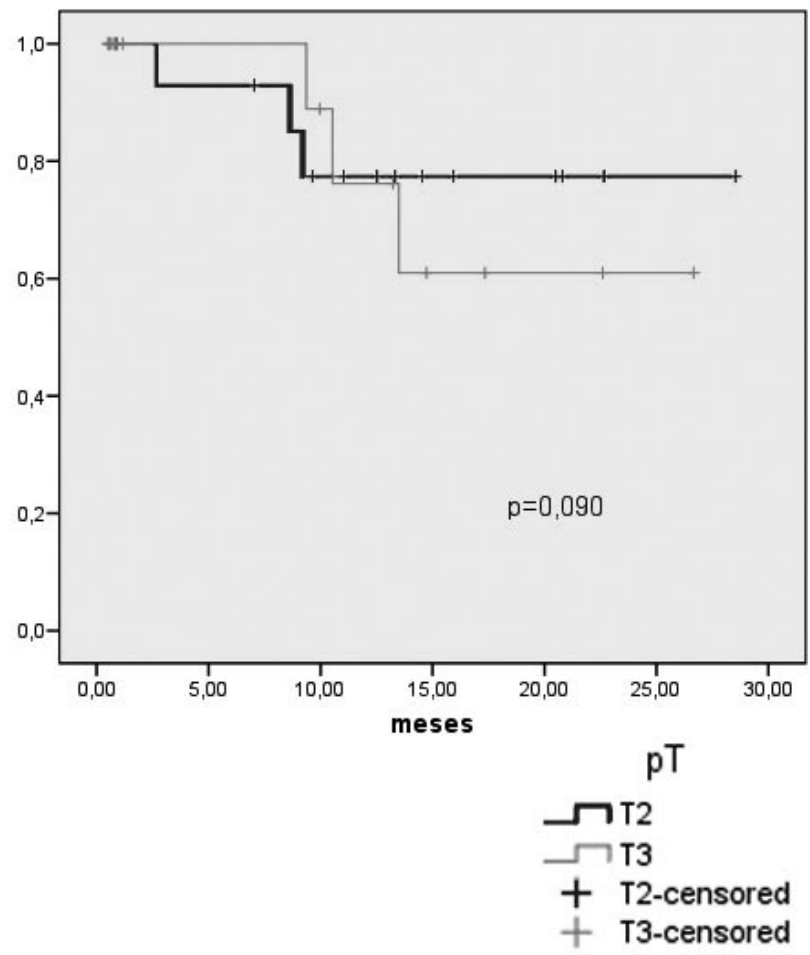

FIGURA 3. Supervivencia global por estadios. abdomen abierto durante este procedimiento de larga duración se evita gran parte de la pérdida insensible de líquidos que ello supone (25). El realizar el tiempo de exéresis por vía laparoscópica, evitando la manipulación intestinal excesiva y sólo utilizar una incisión limitada en tamaño y tiempo de retracción músculo aponeurótica contribuyen a esta mejor recuperación (10).

Finalmente, una ventaja adicional y creemos que relevante de este abordaje es que sirve como ejercicio de práctica de disección pélvica. En casos seleccionados con poca carga tumoral, el cirujano que aprende laparoscopia puede ejercitarse en la disección de los espacios pélvicos. Desde el punto de vista anatómico y de resección quirúrgica es una técnica que requiere menos precisión que la prostatectomía radical laparoscópica y permite el ensayo de algunos pasos de esa técnica sin arriesgar los resultados oncológicos o funcionales.

Obviamente la técnica laparoscópica no esta libre de inconvenientes. Estos suelen derivarse de la manipulación de una pieza de gran tamaño, que se pueden soslayar con una técnica quirúrgica reglada, evitando la movilización precoz del espécimen. Existe una incisión de laparotomía obligada para la extracción de un espécimen de gran tamaño, aunque excepcionalmente puede obviarse cuando la pieza se puede extraer por la vagina o el recto. Este inconveniente lo aprovechamos para la realización de la derivación urinaria de forma extracorpórea, y además permite la ampliación de la linfadenectomía en su límite craneal. En cuanto al control oncológico tras la manipulación laparoscópica de la pieza, se requieren series con mayor seguimiento para valorar si realmente existe un riesgo añadido de sembrar células. Para intentar prevenir al máximo este fenómeno es de vital importancia la movilización gentil del espécimen, el clipaje de los uréteres y de la uretra, que solemos hacer con Hemolok $(8)$ y el embolsado precoz de la misma. En nuestra serie, con los T2 no ha habido evidencia de recidiva local que pueda atribuirse a este fenómeno, pero nuestro seguimiento es todavía escaso. Sin embargo, es de destacar el infraestadiaje clínico de la serie, ya que el $48 \%$ de los casos son >=T2 y un $25 \%$ presentaron afectación ganglionar. Pensamos que esto es atribuible a las limitaciones del TC abdominal con contraste junto con la demora entre el estudio y la cirugía radical.

En definitiva, la cistectomía radical laparoscópica debe reproducir los principios de la cirugía abierta y por tanto sus indicaciones son las mismas que las del abordaje clásico: tratamiento del cáncer vesical infiltrante de la capa muscular ( $>=T 2$ ) y del no infiltrante de la capa muscular que no pueda ser 
controlado por técnicas endoscópicas (papilomatosis o del T1 AG ○ Tis sin respuesta al tratamiento endovesical). Debe cumplirse la premisa de que el abordaje laparoscópico no penalice al paciente ni al resultado de la intervención quirúrgica o a las complicaciones derivadas de éste. Finalmente, algunos autores recomiendan restringirlo al T2 como máximo (7) hasta que la experiencia demuestre que permite respetar los mismos principios oncológicos que la cirugía abierta y como mínimo igualar los resultados oncológicos. En nuestro centro, haber ampliado la indicación a todos los tumores tributarios de cistectomía radical no nos ha supuesto peores resultados.

\section{CONCLUSIONES}

La cistectomía radical laparoscópica es una técnica factible que ofrece ventajas. Permite una exéresis con un menor sangrado y un postoperatorio más llevadero. Estudios aleatorizados deberían demostrar estas ventajas para confirmar si puede llegar a ser la técnica de elección. La realización de la derivación urinaria por la laparotomía, obligada para la extracción de la pieza quirúrgica, optimiza los resultados de la derivación y el tiempo quirúrgico total sin reducir los beneficios de la exéresis laparoscópica.

\section{BIBLIOGRAFÍA y LECTURAS RECOMENDADAS (*lectura de interés $y^{* *}$ lectura fundamental)}

*1. PARRA, R.O.; ANDRUS, C.H.; JONES, J.P. y cols.: "Laparoscopic cystectomy: Initial report on a new treatment for the retained bladder". J. Urol., 148: 1140, 1992.

2. SANCHEZ DE BADAJOZ, E.; GALLEGO PERALES, J.L.; RECHE ROSADO, A. y cols.: "Radical cystectomy and laparoscopic ileal conduit". Arch. Esp. Urol., 46: 621, 1993.

3. HUANG, G.J.; STEIN, J.P.: "Open radical cystectomy with lymphadenectomy remains the treatment of choice for invasive bladder cancer". Curr. Opin. Urol., 17: 369, 2007.

4. TURK, I.; DEGER, S.; WINKELMANN, B. y cols.: "Laparoscopic radical cystectomy with continent urinary diversion (rectal sigmoid pouch) performed completely intracorporeally: The initial 5 cases". J. Urol., 165: 1863, 2001.

5. EL-TABEY, N.A.; SHOMA, A.M.: "Port site metastases after robot-assisted laparoscopic radical cystectomy". Urology, 66: 1110, 2005.

6. PORPIGLIA, F.; RENARD, J.; BILLIA, M. y cols.: "Fast and safe closing of urethra during laparoscopic radical cystectomy". J. Endourol., 20: 651, 2006.

VAN VELTHOVEN, R.F.; PIECHAUD, T.: "Lapa-

7. roscopic radical cystectomy with ileal conduit diversion". Curr. Urol. Rep., 6: 93, 2005.

8. CECCHINI, L.; ORSOLA, A.; MOROTE, J.: "Cistectomía Radical Laparoscópica: Perspectivas His- tóricas, Presente y Futuro de la Cirugía Laparoscópica”. Ed. Evidencia Médica, 22: 163, 2008.

9. CECCHINI, L.; ORSOLA, A.; MOROTE, J.: "Cistectomía radical laparoscópica: ¿Es necesaria?”. Laparoscopia, Editorial Médica Panamericana, vol. 4, 2007.

**10. CATHELINEAU, X.; ARROYO, C.; ROZET, F. y cols.: "Laparoscopic assisted radical cystectomy: The Montsouris experience after 84 cases". Eur. Urol., 47: 780, 2005.

*11. SIMONATO, A.; GREGORI, A.; LISSIANI, A. y cols.: "Laparoscopic radical cystoprostatectomy: A technique illustrated step by step". Eur. Urol., 44: $132,2003$.

*12. ARON, M.; COLUMBO, J.R. Jr.; HABER, G.P. y cols.: "Laparoscopic radical cystectomy". BJU Int., 100: 455, 2007

*13. HASSON, H.M.: "A modified instrument and method for laparoscopy”. Am. J. Obstet. Gynecol., 110: $886,1971$.

14. HERR, H.W.; BOCHNER, B.H.; DALBAGNI, G. y cols.: "Impact of the number of lymph nodes retrieved on outcome in patients with muscle invasive bladder cancer". J. Urol., 167: 1295, 2002.

15. DENEWER, A.; KOTB, S.; HUSSEIN, O. y cols.: "Laparoscopic assisted cystectomy and lymphadenectomy for bladder cancer: Initial experience". World J. Surg., 23: 608, 1999.

16. ABDEL-HAKIM, A.M.; BASSIOUNY, F.; ABDEL AZIM, M.S. y cols.: "Laparoscopic radical cystectomy with orthotopic neobladder". J. Endourol., 16: 377, 2002.

17. MATIN, S.F.; GILL, I.S.: "Laparoscopic radical cystectomy with urinary diversion: Completely intracorporeal technique". J. Endourol., 16: 335, 2002.

18. GUAZZONI, G.; CESTARI, A.; COLOMBO, R. y cols.: "Laparoscopic nerve- and seminal-sparing cystectomy with orthotopic ileal neobladder: The first three cases". Eur. Urol., 44: 567, 2003.

*19. HEMAL, A.K.; KOLLA, S.B.: "Comparison of laparoscopic and open radical cystoprostatectomy for localized bladder cancer with 3-year oncological followup: A single surgeon experience". J. Urol., 178: 2340, 2007.

**20. HABER, G.P.; GILL, I.S.: "Laparoscopic radical cystectomy for cancer: Oncological outcomes at up to 5 years". BJU Int., 100: 137, 2007.

21. FARNHAM, S.B.; WEBSTER, T.M.; HERRELL, S.D. y cols.: "Intraoperative blood loss and transfusion requirements for robotic-assisted radical prostatectomy versus radical retropubic prostatectomy". Urology, 67: 360, 2006.

22. SARANCHUK, J.W.; SAVAGE, S.J.: "Laparoscopic radical nephrectomy: Current status". BJU Int., 95: 21, 2005.

23. DUNN, M.D.; PORTIS, A.J.; SHALHAV, A.L. y cols.: "Laparoscopic versus open radical nephrectomy: A 9 year experience". J. Urol., 164: 1153, 2000.

24. RAVENTOS, C.; CECCHINI, L.; TRILLA, E. y cols.: "Prostatectomía radical laparoscópica versus abierta". Actas Urológicas Españolas, 31: 141, 2007.

25. UKIMURA, O.; MOINZADEH, A.; GILL, I.S.: "Laparoscopic radical cystectomy and urinary diversion”. Curr. Urol. Rep., 6: 118, 2005. 